Wright State University

CORE Scholar

$11-1-1987$

\title{
Equivalence of Donor and Acceptor Fits To Temperature- Dependent Hall Data: General Case
}

David C. Look

Wright State University - Main Campus, david.look@wright.edu

Follow this and additional works at: https://corescholar.libraries.wright.edu/physics

Part of the Physics Commons

\section{Repository Citation}

Look, D. C. (1987). Equivalence of Donor and Acceptor Fits To Temperature-Dependent Hall Data: General Case. Journal of Applied Physics, 62 (9), 3998-3999.

https://corescholar.libraries.wright.edu/physics/110

This Article is brought to you for free and open access by the Physics at CORE Scholar. It has been accepted for inclusion in Physics Faculty Publications by an authorized administrator of CORE Scholar. For more information, please contact library-corescholar@wright.edu. 


\author{
D. C. Look \\ University Research Center, Wright State University, Dayton, Ohio 45435
}

(Received 2 April 1987; accepted for publication 28 July 1987)

\begin{abstract}
Recently, it was shown that the usual statistical-mechanical formulation used to fit carrier concentration versus temperature data cannot distinguish between the donor or acceptor nature of one single-charge-state center. Here we generalize that result to include any number of donor and acceptor centers, of arbitrary charge multiplicity, and also show how that by fitting one particular case (e.g., every center assumed to be a donor), all of the other possible cases can be immediately solved by inspection.
\end{abstract}

A common method to determine donor and acceptor concentrations and energy levels in a semiconductor is to fit temperature-dependent Hall-effect data with the chargebalance equation, derived from statistical mechanics. ${ }^{1,2}$ Very recently, we showed ${ }^{3}$ that such a fit cannot distinguish between the donor or acceptor nature of one single-chargestate center, even though the charge-balance equations for the donor and acceptor cases appear to be different. The main purpose of the present work, which should be considered an addendum to Ref. 3, is to give a general proof of the equivalence of donor and acceptor fits for all cases, including multicharge centers. It is shown in Ref. 4 that the generalized "charge-balance" equation for arbitrary donor and acceptor species (each designated by $k$ ) is

$$
n=p+\sum_{\substack{k, l, m \\ l_{k}=0 \text { for } A}}\left(l_{k}-l\right) n_{k l m},
$$

where $l_{k}$ is the number of electrons ionizable from the neutral state ( $l_{k}=0$ if center $k$ is a pure acceptor), $l$ ranges from zero to the maximum change of electron occupation, and

$$
\begin{aligned}
n_{k l m}= & N_{k}\left(1+\sum_{l^{\prime}, m^{\prime} \neq l, m} \frac{g_{k l^{\prime} m^{\prime}}}{g_{k l m}}\right. \\
& \left.\times \exp \left\{\left[\epsilon_{k l m}-\epsilon_{k l^{\prime} m^{\prime}}-\left(l-l^{\prime}\right) \epsilon_{F}\right] / k_{B} T\right\}\right)^{-1} .
\end{aligned}
$$

The index $m$ denotes excited states, and the summation restriction is that $l \neq l^{\prime}$ and $m \neq m^{\prime}$ at the same time. Note that the only asymmetry between donors and acceptors in Eq. (1a) is the factor $l_{k}$, which is the total number of electrons that donor center $k$ may release from its neutral state (with the final electron still requiring less than band-gap energy for ionization). For pure donors, the summation index $l$ will range from 0 to $l_{k}$, while for pure acceptors, $l_{k}=0$, and $l$ ranges from 0 to the number of electrons that acceptor center $k$ may trap onto its neutral state. Consider, e.g., a triple donor, for which $l_{k}=3$ and $l=0,1,2,3$ and compare that with a triple acceptor, for which $l_{k}=0$ and $l=0,1,2,3$. To make the acceptor appear as a donor in the last term of Eq. (1a), we simply define an acceptor $l_{k}$ to equal the maximum $l$ for the acceptor, and then add and subtract equal terms in Eq. (1a). Formally,

$$
\begin{aligned}
n & =p+\sum_{\substack{k, l, m \\
l_{k}=0 \text { for } A}}\left(l_{k}-l\right) n_{k l m} \\
& =p+\sum_{\substack{k, l, m \\
\text { all } A, D}}\left(l_{k}-l\right) n_{k l m}-\sum_{k, l, m} l_{k} n_{k l m} \\
& =p+\sum_{\substack{k, l, m \\
\text { all } A, D}}\left(l_{k}-l\right) n_{k l m}-\sum_{k} l_{k} N_{k},
\end{aligned}
$$

where now the only asymmetry between donors and acceptors is in the last term of Eq. (2). But the last term is temperature independent and thus will simply add to the temperature-independent part of the next-to-last term, which arises from the "shallow" centers, both donors $\left(N_{\mathrm{DS}}\right)$ and acceptors ( $N_{\text {AS }}$ ), whose occupations do not change over the temperature range of the experiment. However, the major parameters of an $n$-vs- $T$ fit, namely, the concentrations, energy levels, and degeneracy factors of the principal centers, come from the temperature-dependent parts of the next-tolast term and are not affected by the absence or presence of the last term. [To show this, we simply need to take a temperature derivative of Eq. (2) and fit that equation, instead.] Then, since the next-to-last term is independent of any particular center's donor or acceptor nature, the proof is complete.

To illustrate, we consider a case in which there are four single-charge-state $\left(l_{k}=1\right)$ centers, $C_{1}, C_{2}, C_{3}$, and $C_{4}$. Over the temperature range of interest, $C_{2}$ and $C_{3}$ have variable occupation factors, whereas $C_{1}$ is a donor of concentration $N_{\text {DS }}$, lying well above $\epsilon_{F}$ at all temperatures, and $C_{4}$ is an acceptor of concentration $N_{\text {AS }}$, lying well below $\epsilon_{F}$. Then, with the excited-state index $m$ suppressed, Eq. (2) becomes

$$
\begin{aligned}
n & =p+\sum_{k=2,3} n_{k 0}+K \\
& =p+\sum_{k=2,3} \frac{N_{k}}{1+\left(g_{1} / g_{0}\right)_{k} e^{-\alpha_{k} / k_{B}} e^{\left(E_{k 0}-E_{F}\right) / k_{B} T}}+K,
\end{aligned}
$$

where now the energies are defined with respect to the conduction band, and $\alpha_{k}$ is defined by $E_{k}=E_{k 0}-\alpha_{k} T$. The fitted parameters are $N_{k}, E_{k 0},\left(g_{1} / g_{0}\right)_{k} \exp \left(-\alpha_{k} / k_{B}\right)$, and $K$, for $k=2,3$. Note that the first three parameters are inde- 
TABLE I. Interpretation of constant $K$ in Eq. (6) $\left(N_{1} \equiv N_{\mathrm{DS}}\right.$ and $N_{4}$ $\equiv N_{\text {AS }}$ ).

\begin{tabular}{lll}
\hline \hline$C_{2}$ & $C_{3}$ & \multicolumn{1}{c}{$K$} \\
\hline $\mathrm{D}$ & $\mathrm{D}$ & $N_{\text {Ds }}-N_{\text {As }}$ \\
$\mathrm{D}$ & $\mathrm{A}$ & $N_{\text {Ds }}-N_{\text {As }}-N_{3}$ \\
$\mathrm{~A}$ & $\mathrm{D}$ & $N_{\mathrm{DS}}-N_{\mathrm{As}}-N_{2}$ \\
$\mathrm{~A}$ & $\mathrm{~A}$ & $N_{\mathrm{DS}}-N_{\text {As }}-N_{2}-N_{3}$ \\
\hline
\end{tabular}

pendent of the donor or acceptor nature of $C_{2}$ and $C_{3}$. However, the definition of $K$ changes, as shown in Table $\mathrm{I}$, and since $N_{2}$ and $N_{3}$ are already determined from the fit, $N_{\text {Ds }}-N_{\text {As }}$ can be immediately calculated for each case.

This work was carried out in the Avionics Laboratory, Wright-Patterson Air Force Base, under Contract No. F33615-86-C-1062.
'J. S. Blakemore, Semiconductor Statistics (Dover, New York, 1983). ${ }^{2}$ D. C. Look, Phys. Rev. B 24, 5852 ( 1981 ).

${ }^{3}$ D. C. Look and J. R. Sizelove, J. Appl. Phys. 61, 1650 (1987).

${ }^{4}$ D. C. Look, in Semiconductors and Semimetals, edited by R. K. Willardson and A. C. Beer (Academic, New York, 1983), Vol. 19, p. 162.

\title{
Effect of interface structure on photoluminescence of InGaAs/GaAs pseudomorphic single quantum wells
}

\author{
R. L. S. Devine and W. T. Moore \\ National Research Council of Canada, Division of Physics, Ottawa KIA OR6, Canada
}

(Received 23 February 1987; accepted for publication 29 June 1987)

\begin{abstract}
Photoluminescence studies have been carried out on InGaAs pseudomorphic single quantum wells grown on GaAs by molecular beam epitaxy. Linewidths as narrow as $2.0 \mathrm{meV}$ have been observed. The spectra typically consist of two peaks which on certain samples can be explained by intrawell thickness variations of one monolayer. On other samples the results are more consistent with exciton trapping at islands having a smaller lateral extent than the exciton Bohr radius.
\end{abstract}

In recent years there has been considerable interest in the use of photoluminescence (PL) as a means of investigating the interface quality of quantum well $(\mathrm{QW})$ structures. One aspect of this work has been the demonstration of multiple peaks in the PL spectrum due to intrawell thickness variations in GaAs/AlGaAs QW's. ${ }^{1-4}$ These are observed when the diameters of the stepped regions are larger than the diameter of the exciton. Interruption of the growth at the interface, leading to a smoothing of the surface, has been shown to give this condition. In QW's grown without interruption, a single wide peak is generally observed. This peak is often Stoke's shifted to lower energies due to trapping of the excitons at regions where the $\mathrm{QW}$ is wider, but the lateral extent is smaller than the exciton Bohr radius.

The $\mathrm{InGaAs} / \mathrm{GaAs}$ strained-layer materials system is coming under increasing investigation due to its promising device potential in both optoelectronics and in field effect transistor structures. ${ }^{5}$ InGaAs pseudomorphic QW's have generally shown inferior linewidths compared to equivalent $\mathrm{GaAs} / \mathrm{AlGaAs}$ structures and have therefore been of limited use for interface studies. In principle, the absence of the relatively rough AlGaAs surface in these structures should eliminate the problem of asymmetric interfaces. However, other effects, such as indium segregation, could possibly complicate the situation. In this communication we present
PL spectra from pseudomorphic InGaAs single QW's with linewidths narrow enough such that trapping effects are clearly visible.

The samples were grown by molecular-beam epitaxy (MBE) on (100)-oriented semi-insulating GaAs substrates. Both $\mathrm{As}_{4}$ and $\mathrm{As}_{2}$ (from a cracker cell) were employed, with typical As/Ga beam equivalent pressure ratios of 10 and 4 , respectively. Indium-free mounting was used for all the substrates. The indium concentration $\left(x_{1 \mathrm{n}}\right)$ in the QW's is typically $15 \%-20 \%$. In all cases a GaAs buffer layer of at least $1 \mu \mathrm{m}$ was deposited before the $\mathrm{QW}$, followed by a $600 \AA$ capping layer. The buffer layer and $\mathrm{QW}$ structure were grown at 640 and $520^{\circ} \mathrm{C}$, respectively. The growth rate was obtained from thick calibration layers. The luminescence was obtained using the conventional lock-in technique with a 0.5-m Jarrel-Ash monochromator. The excitation source was a $\mathrm{HeNe}$ laser with a typical excitation intensity $\sim 1 \mathrm{~W}$ $\mathrm{cm}^{-2}$. All measurements were carried out at $5 \mathrm{~K}$ using a continuous flow cryostat. The spectra obtained were curve fitted using the Levenberg-Marquardt $\operatorname{method}^{6}$ and the best fit chosen by minimizing a chi-square merit function.

Figure 1 shows the PL spectrum at $\sim 5 \mathrm{~K}$ obtained from a QW with $L_{z}=73 \AA$ and $x_{1 \mathrm{n}} \sim 0.17$, together with the spectrum generated by the curve-fitting procedure. The experimental curve is seen to be closely fitted by a superposition of 